Systemic lupus erythematosus (SLE) is a clinically heterogeneous multi-system disease, that is characterised by the presence of autoantibodies directed against nuclear antigens. The most common manifestations include rash, arthritis, fatigue, but also anaemia, thrombocytopenia, nephritis and neurologic symptoms. Despite enormous improvements in prognosis since the introduction of immunosuppressive drugs, SLE continues to have a significant impact on the mortality and morbidity of those affected.

The aim of this project was to understand the prevalence, morbidity and outcome associated with lupus nephritis at our auto-immunity diseases centre. Clinical records of 128 patients treated between January 1993 and December 2016 were read, and national registry of Auto-immune diseases was consulted in order to characterise the Lupus cohort. Treatment was assessed and pre and post treatment biopsies were reviewed by WHO classification.

From all 137 patients assessed, average age was 49, with 93\% of all patients being females. The most frequent clinical criteria were malar rash in 72\%, arthritis in 51\%, hematologic disturbances in 43\%, from which the most frequent was lymphopenia. Among all patients, $21.2 \%(n=29)$ had clinical and histologic diagnostic criteria for lupus nephritis, with mean age at diagnosis of 34 years old (from 17 to 71). From all biopsies performed, 48\% were classified as grade IV OMS. All patients were treated with glucocorticoids, and 74\% performed induction therapy with Protocol Euro-Lupus, followed by mycophenolate mofetil. ACE inhibitors were used in $95,2 \%$ of all patients. Only two patients worsened and interchanged nephritis class, with one patient achieving kidney failure.

This was an important review for our centre, since our patients presented an elevated proportion of Lupus Nephritis, at a very young age.

\section{PS9:176 SYSTEMIC LUPUS ERYTHEMATOSUS (SLE) PROSPECTIVE OBSERVATIONAL COHORT STUDY (SPOCS) TO CHARACTERISE MODERATE TO SEVERE SLE DISEASE ACTIVITY, TREATMENT, AND OUTCOMES BY TYPE I INTERFERON GENE SIGNATURE}

${ }^{1}$ ER Hammond, ${ }^{1} \mathrm{R}$ Tummala, ${ }^{2} \mathrm{~A}$ Berglind, ${ }^{1} \mathrm{~B}$ Desta, ${ }^{3} \mathrm{H}$ Nab. ${ }^{1}$ AstraZeneca, Gaithersburg, MD, USA; ${ }^{2}$ AstraZeneca, Gothenburg, Sweden; ${ }^{3}$ AstraZeneca, Cambridge, UK

\subsection{6/lupus-2018-abstract.219}

Purpose Type I interferon (IFN) plays a major role in SLE pathogenesis. However, limited information exists about type I IFN gene signature (IFNGS) associations with disease severity and activity, health-related quality of life, and outcomes for the general population of patients with moderate to severe SLE receiving standard-of-care treatment.

Methods Initiated in June 2017, SPOCS is an international, multicenter cohort of 1500 patients with moderate to severe SLE evaluated biannually during a 3 year follow-up period. Participating countries include Canada, United States, France, Germany, Italy, Spain, United Kingdom, and Australia. SPOCS will systematically describe the comprehensive patient journey, including clinical features, disease progression and treatment, outcomes, health status, and health care resource utilisation, for a general population of patients with moderate to severe SLE (table 1).

Association of type I IFNGS expression with these elements will be assessed. The study includes 2 year enrollment and

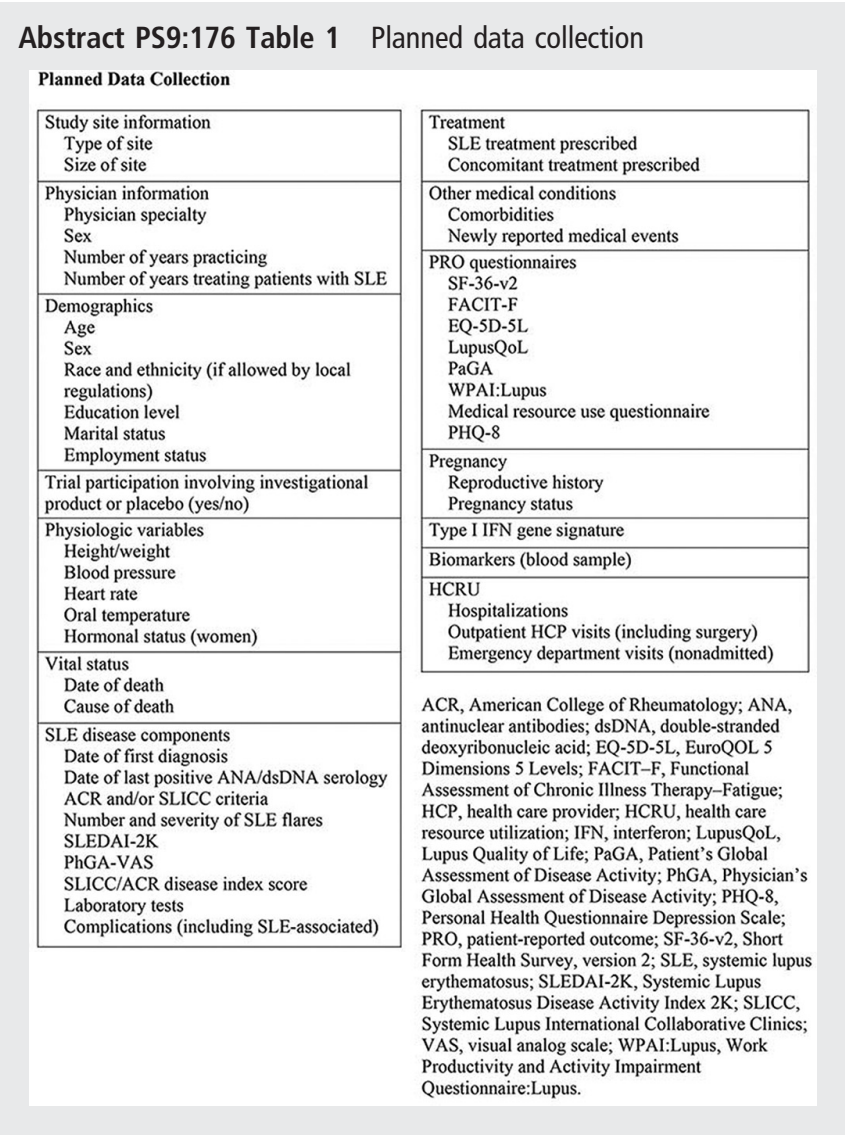

3 year follow-up periods for each patient. Patients $(\geq 18$ years old) with a physician diagnosis that meets ACR or SLICC SLE criteria will be included. Additional study entry requirements include moderate to severe SLE as defined by a modified SLEDAI- $2 \mathrm{~K}$ score $\geq 4$ or SLEDAI- $2 \mathrm{~K}$ score $\geq 6$, $\geq 6$ month treatment duration for active SLE with systemic SLE treatment beyond NSAIDs and analgesics, and current or historic serology of ANA or dsDNA. Exclusion criteria include enrollment in interventional trials involving investigational agents or active, severe, biopsy-confirmed class III or class IV \pm class V $\mathrm{LN}$ and/or urine protein:creatinine ratio $>1 \mathrm{mg} / \mathrm{mg}$. Patients will be followed as per local routine clinical practice.

Results First patient recruited was achieved in June 2017, and the last patient out is anticipated for Q2 2022. Data collection, which will include use of electronic case report forms and patient-reported outcomes, will take place at biannual study visits. Distribution of type I IFNGS (test-high vs testlow) will be determined, and any association with patient outcomes will be evaluated.

Conclusion SPOCS will provide important information about possible associations of type I IFNGS with disease characteristics and outcomes for patients with moderate to severe SLE.

\section{PS9:177 LUPUS NEPHRITIS - CLINICOPATHOLOGICAL CORRELATION AND RENAL OUTCOME}

${ }^{1} \mathrm{R}$ Rajasekharan Nair, ${ }^{1} \mathrm{P}$ Harish, ${ }^{2} \mathrm{~S}$ Seethalekshmy, ${ }^{1} \mathrm{G}$ Kurian, ${ }^{1} \mathrm{~A}$ Mathew, ${ }^{1} \mathrm{Z}$ Paul, S Sreedharan 1. 'Dept of Nephrology, Amrita institute of Medical Sciences, Kochi, Kerala, India; ${ }^{2}$ Dept of Pathology, Amrita institute of Medical Sciences, Kochi, Kerala, India

10.1136/lupus-2018-abstract.220 